\title{
Equilibrium fluctuations of additive functionals of zero-range models
}

Cédric Bernardin, Patrícia Gonçalves and Sunder Sethuraman

\begin{abstract}
For mean-zero and asymmetric zero-range processes on $\mathbb{Z}^{d}$, the fluctuations of additive functionals starting from an invariant measure are considered. Under certain assumptions, we establish when the fluctuations are diffusive and satisfy functional central limit theorems. These results complement those for symmetric zero-range systems and also those for simple exclusion models already in the literature.
\end{abstract}

\section{Introduction and model assumptions}

We consider zero-range processes which follow a collection of random walks interacting on $\mathbb{Z}^{d}$ in the following way: When there are $k$ particles at a location $x$, one of them displaces by $y$ with rate $[g(k) / k] p(y)$. Here, $g:\{0,1,2, \ldots\} \rightarrow \mathbb{R}_{+}$is a prescribed function such that $g(0)=0$ and $g(k)>0$ for $k \geq 1$, and $p$ is a jump probability on $\mathbb{Z}^{d}$. Another way to think of the process is that each location $x \in \mathbb{Z}^{d}$ has a clock which rings at rate $g(k)$ where $k$ is the particle number at $x$. Once the

Cédric Bernardin

Université de Nice Sophia-Antipolis, Laboratoire J.A. Dieudonné, UMR CNRS 7351, Parc Valrose, 06108 Nice cedex 02- France e-mail: cbernard@unice.fr

Patrícia Gonçalves

PUC-RIO, Departamento de Matemática, Rua Marquês de São Vicente, no. 225, 22453-900,

Gávea, Rio de Janeiro, Brazil

and

CMAT, Centro de Matemática da Universidade do Minho, Campus de Gualtar, 4710-057 Braga, Portugal.

e-mail: patricia@mat.puc-rio.br

Sunder Sethuraman

Mathematics, University of Arizona, 617 N. Santa Rita Ave., Tucson, AZ 85721. e-mail: sethuram@math.arizona.edu 
clock rings at $x$, a particle selected at random displaces by $y$ with probability $p(y)$. This well-studied model has been used in the modeling of traffic, queues, granular media, fluids, etc. [2], and also includes the case of independent random walks when $g(k) \equiv k$.

In this note, we study the equilibrium fluctuations of additive functionals in a class of zero-range processes, namely those which are 'asymmetric', 'attractive', and for which a 'spectral gap' estimate holds. When the model is 'symmetric', the fluctuation behaviors are found in [15] and [9]. Also, part of the motivation of this note is to complement the much detailed work on fluctuations in simple exclusion processes (cf. Chapter 5 of [6]), as much less is known for zero-range systems. The arguments make use of a combination of techniques in the literature. We now define more carefully the model and related terms.

\subsection{Jump rates and construction.}

We will assume that the function $g$ allows motion: $g(0)=0$ and $g(k)>0$ for $k \geq 1$ and the following two conditions.

(LIP) There is a constant $K$ such that $|g(k+1)-g(k)|<K$ for $k \geq 0$

(INC) $g$ is increasing: $g(k+1) \geq g(k)$ for $k \geq 0$.

Condition (LIP) is usually assumed in order to construct the process on an infinite lattice. However, although also often assumed, (INC) is a more technical condition which makes avaliable a certain 'basic coupling' that we will use later.

Assume also that $p$ is such that the symmetrization $s(x)=(p(x)+p(-x)) / 2$ is irreducible and

(FR) $\quad p$ is finite-range: There is an $R$ such that $p(z)=0$ for $|z|>R$.

Here, $|z|=\max \left\{\left|z_{i}\right|: i=1, \ldots, d\right\}$. The assumption (FR) might presumably be relaxed in favor of a $p$ with rapidly diminishing tail behavior, although we do not consider this case or when $p$ might have heavy tails where certainly the results would differ.

Under these conditions, more restrictive than necessary, the zero-range system $\eta_{t}=\left\{\eta_{t}(x): x \in \mathbb{Z}^{d}\right\}$ can be constructed as a Markov process on the state space $\Omega:=\mathbb{N}_{0}^{\mathbb{Z}^{d}}$ with generator $L$ whose action on local functions is given by

$$
L f(\eta)=\sum_{x, y \in \mathbb{Z}^{d}} g(\eta(x)) p(y)\left[f\left(\eta^{x, x+y}\right)-f(\eta)\right] .
$$

Here, $\eta_{t}(x)$ is the occupation number at $x$ at time $t, \eta^{x, x+y}$ is the state obtained from $\eta$ by decreasing and increasing the occupation numbers at $x$ and $y$ by one respectively, and a local function is one which depends only on a finite number of coordinates $\{\eta(z)\}$. See [1] for the construction and weakening of the assumptions. 
We will say that the system is symmetric, mean-zero asymmetric or asymmetric with drift if $p$ satisfies $p(z)=p(-z), \sum_{z \in \mathbb{Z}^{d}} z p(z)=0$ but $p$ is not symmetric, and $\sum_{z \in \mathbb{Z}^{d}} z p(z) \neq 0$ respectively.

\subsection{Invariant measures.}

Part of the appeal of zero-range processes is that they possess a family of invariant measures which are fairly explicit product measures. For $\alpha \geq 0$, define

$$
z(\alpha):=\sum_{k \geq 0} \frac{\alpha^{k}}{g(k) !}
$$

where $g(k) !=g(1) \cdots g(k)$ for $k \geq 1$ and $g(0) !=1$. Let $\alpha^{*}$ be the radius of convergence of this power series and notice that $z$ increases on $\left[0, \alpha^{*}\right)$. Fix $0 \leq \alpha<\alpha^{*}$ and let $\bar{v}_{\alpha}$ be the product measure on $\mathbb{N}^{\mathbb{Z}}$ whose marginal at the site $x$ is given by

$$
\bar{v}_{\alpha}\{\eta: \eta(x)=k\}=\left\{\begin{array}{r}
\frac{1}{z(\alpha)} \frac{\alpha^{k}}{g(k) !} \text { when } k \geq 1 \\
\frac{1}{z(\alpha)} \text { when } k=0 .
\end{array}\right.
$$

We may reparametrize these measures in terms of the 'density'. Let $\rho(\alpha):=$ $E_{\bar{v}_{\alpha}}[\eta(0)]=\alpha Z^{\prime}(\alpha) / Z(\alpha)$. By computing the derivative, we obtain that $\rho(\alpha)$ is strictly increasing on $\left[0, \alpha^{*}\right)$. Then, let $\alpha(\cdot)$ denote its inverse. Now, we define

$$
v_{\rho}(\cdot):=\bar{v}_{\alpha(\rho)}(\cdot),
$$

so that $\left\{v_{\rho}: 0 \leq \rho<\rho^{*}\right\}$ is a family of invariant measures parameterized by the density. Here, $\rho^{*}=\lim _{\alpha \uparrow \alpha^{*}} \rho(\alpha)$, which may be finite or infinite depending on whether $\lim _{\alpha \rightarrow \alpha^{*}} Z(\alpha)$ converges or diverges. In this notation, $\alpha(\rho)=E_{v_{\rho}}[g(\eta(0)]$ is a 'fugacity' parameter.

One may check that the measures $\left\{v_{\rho}: 0 \leq \rho<\rho^{*}\right\}$ are invariant for the zerorange process [1]. Moreover, we remark, by the construction in [14], which extends the construction in [1] to an $L^{2}\left(v_{\rho}\right)$ process, we have that $L$ is a Markov $L^{2}\left(v_{\rho}\right)$ generator whose core can be taken as the space of all local $L^{2}\left(v_{\rho}\right)$ functions. Also, one may compute that the adjoint $L^{*}$ is the zero-range process with jump probability $p^{*}(z)=p(-z)$ for $z \in \mathbb{Z}^{d}$. The operator

$$
S=\left(L+L^{*}\right) / 2
$$

may be seen as the generator for the symmetrized process with jump law $s$. In particular, when $p$ is symmetric, the process is reversible with respect to $v_{\rho}$ with generator $L=L^{*}=S=\left(L+L^{*}\right) / 2$. 
It is also known that the family $\left\{v_{\rho}: \rho<\rho^{*}\right\}$ are all extremal invariant measures, and hence when the process is started from one of them, the system will be ergodic with respect to time shifts. Let us now fix one of these invariant measures $v_{\rho}$ throughout the article.

\subsection{Spectral gap.}

To state results, we will need to detail the spectral gap properties of the system. For $\ell \geq 1$, let $\Lambda_{\ell}=\left\{x \in \mathbb{Z}^{d}:|x| \leq \ell\right\}$. Consider the 'symmetrized' process restricted to $\Lambda_{\ell}$ with generator

$$
S_{\ell} f(\eta)=\sum_{\substack{|x| \leq \ell,|y|=1 \\|x+y| \leq \ell}} s(y) g(\eta(x))\left[f\left(\eta^{x, x+y}\right)-f(\eta)\right] .
$$

Given the number particles in $\Lambda_{\ell}$, say $\sum_{|x| \leq \ell} \eta(x)=M$, one can verify that the process is a reversible finite-state Markov chain with unique invariant measure $v_{\rho}\left(\cdot \mid \sum_{|x| \leq \ell} \eta(x)=M\right.$ ) (which does not depend on $\rho$ ). Hence, since the chain is irreducible, there is a gap in the spectrum of $S_{\ell}$ between the eigenvalue 0 and the next one which is strictly negative. Let $W(M, \ell)$ be the reciprocal of the absolute value of this 'spectral gap'. Also, $W(M, \ell)$ can be captured as the smallest constant $c$ such that the Poincaré inequality, $E_{v_{\rho}}\left[f^{2}\right] \leq c E_{v_{\rho}}\left[f\left(-S_{\ell} f\right)\right]$, holds for all local mean-zero function $f, E_{v_{\rho}}[f]=0$.

We will assume that the following estimate holds:

(G) There is a constant $C=C(\rho)$ such that for all $\ell \geq 1$, we have

$$
E_{v_{\rho}}\left[W^{2}\left(\sum_{|x| \leq \ell} \eta(x), \ell\right)\right] \leq C(\rho) \ell^{4}
$$

Such an estimate is a further condition on $g$ and $p$ and holds in a number of cases. Usually, one tries to bound the spectral gap for the corresponding nearest-neighbor process. Given assumption (FR), by comparing the associated Poincaré inequalities, the order of $W(M, \ell)$, asymptotically in $\ell$, with respect to $S_{\ell}$ and the nearest-neighbor version will be the same.

- If $g$ is not too different from the independent case, that is $g(x) \equiv x$, for which the gap is of order $O\left(\ell^{-2}\right)$ uniform in $x$, one expects similar behavior as for a single particle. This has been proved for $d \geq 1$ in [7] under assumptions (LIP) and

(U) There exists $x_{0} \geq 1$ and $\varepsilon_{0}>0$ such that $g\left(x+x_{0}\right)-g(x) \geq \varepsilon_{0}$ for all $x \geq 0$.

- If $g$ is sublinear, that is $g(x)=x^{\gamma}$ for $0<\gamma<1$, then it has been shown that the spectral gap depends on the number of particles $k$, namely the gap for $d \geq 1$ is $O\left((1+\beta)^{-\gamma} \ell^{-2}\right)$ where $\beta=k /(2 \ell+1)^{d}[11]$. 
- If $g(x)=\mathbf{1}_{x \geq 1}$, then it has been shown in $d \geq 1$ that the gap is $O\left((1+\beta)^{-2} \ell^{-2}\right)$ where $\beta=k /(2 \ell+1)^{d}$ [10]. In $d=1$, this is true because of the connection between the zero-range and simple exclusion processes for which the gap estimate is well-known [12]: The number of spaces between consecutive particles in simple exclusion correspond to the number of particles in the zero-range process.

In all these cases, $(\mathrm{G})$ follows readily by straightforward moment calculations.

\subsection{Attractivity.}

A main technical tool we will use is the 'basic coupling' for interacting particle systems. Such a coupling is available for zero-range processes when $g$ is an increasing function.

Namely, consider two zero-range systems $\eta_{t}^{0}$ and $\eta_{t}^{1}$ started from initial configurations $\eta_{0}^{0}=\eta^{0}$ and $\eta_{0}^{1}=\eta^{1}$ such that $\eta^{0}(x) \leq \eta^{1}(x)$ for all $x \in \mathbb{Z}^{d}$. Then, one can couple the two systems so that whenever a ' 0 ' particle moves, a corresponding ' 1 ' particle makes the same jump. That is, a particle at $x$ in the ' 0 ' and ' 1 ' systems displaces by $y$ with rate $g\left(\eta^{0}(x)\right) p(y)$, and also with rate $\left[g\left(\eta^{1}(x)\right)-g\left(\eta^{0}(x)\right)\right] p(y)$ one of the particles at $x$ in the ' 1 ' system displaces by $y$. In particular, one can write $\eta_{t}^{1}=\eta_{t}^{0}+\xi_{t}$. Here, $\xi_{t}$ counts the 'second-class' or 'discrepancy' particles: $\xi_{t}(x)$ is the number of second-class particles at $x$ at time $t$. See [8] for more details.

\section{Results}

By an additive functional, we mean the time integral of a local function $f$ with respect to the zero-range process:

$$
A_{f}(t)=\int_{0}^{t} f\left(\eta_{s}\right) d s
$$

Since $v_{\rho}$ is extremal, as alluded to earlier, the ergodic theorem captures the law of large numbers behavior

$$
\lim _{t \rightarrow \infty} \frac{1}{t} A_{f}(t)=E_{v_{\rho}}[f]
$$

In this context, the results of this note are on the second-order terms, the fluctuations of $A_{f}(t)$ about its mean. Let $\bar{f}=f-E_{v_{\rho}}[f]$ and

$$
\sigma_{t}^{2}(\rho, f)=E_{v_{\rho}}\left[A_{\bar{f}}(t)^{2}\right]
$$

be the variance at time $t$. One can compute $\sigma_{t}^{2}(\rho, f)$, using stationarity, as follows: 


$$
\begin{aligned}
\sigma_{t}^{2}(\rho, f) & =2 \int_{0}^{t}(t-s) E_{v_{\rho}}\left[\bar{f}\left(\eta_{s}\right) \bar{f}\left(\eta_{0}\right)\right] d s \\
& =2 \int_{0}^{t}(t-s) E_{v_{\rho}}\left[\bar{f}\left(\eta_{0}\right)\left(P_{s} \bar{f}\right)\left(\eta_{0}\right)\right] d s
\end{aligned}
$$

where $P_{t}$ is the semigroup of the process.

One of the main questions is to understand the order of the variance $\sigma_{t}^{2}(\rho, f)$ as $t \uparrow \infty$. Perhaps, surprisingly, this order may or may not be diffusive, that is of order $t$, depending on the function $f$. When the limit exists, we denote

$$
\sigma^{2}(\rho, f):=\lim _{t \uparrow \infty} \frac{1}{t} \sigma_{t}^{2}(\rho, f) .
$$

To explore this point, consider the occupation function $h(\eta)=1(\eta(0) \geq 1)$ which indicates when the origin is occupied. Then, $A_{\bar{h}}(t)$ is the centered occupation time of the origin up to time $t$. When the jump probability $p$ is symmetric, particles tend to stay put more and in $d \leq 2$, when $p$ is recurrent, $A_{h}(t)$ is quite volatile and the variance $\sigma_{t}^{2}(\rho, h)$ is super-diffusive. However, in the transient case, $d \geq 3$, the behavior is more regular and $A_{h}(t)$ has a diffusive variance.

On the other hand, if a function $b$ is somewhat 'smooth', say the difference $b(\eta)=\eta(0)-\eta(1)$ which casts $A_{\bar{b}}(t)$ as the difference of two additive functionals, then one might suspect the variance to be less volatile than under $h$. This is indeed the case, and in all dimensions $d \geq 1, \sigma_{t}^{2}(\rho, b)$ is diffusive.

This phenomenon is summarized by the following result which is Theorem 1.2 of [15]. We say a local function $f$ is admissible if

$$
\underset{t \uparrow \infty}{\limsup } \frac{1}{t} \sigma_{t}^{2}(\rho, f)<\infty
$$

and not admissible otherwise.

\section{Proposition 1 (Theorem 1.2 [15]).}

Suppose assumptions $(L I P),(F R),(G)$ hold, and in addition suppose $p$ is symmetric so that the zero-range process is reversible. Let $f \in L^{4}\left(v_{\rho}\right)$ be a local function supported on coordinates in $\Lambda_{\ell}$. Then, starting from $v_{\rho}, f$ is admissible if and only if

$$
\begin{aligned}
E_{v_{\rho}}[f]=E_{v_{\rho}}\left[f(\eta) \sum_{x \in \Lambda_{\ell}} \eta(x)\right]=E_{v_{\rho}}\left[f(\eta)\left(\sum_{x \in \Lambda_{\ell}} \eta(x)\right)^{2}\right] & =0 \text { in dimension } d=1 \\
E_{v_{\rho}}[f]=E_{v_{\rho}}\left[f(\eta) \sum_{x \in \Lambda_{\ell}} \eta(x)\right] & =0 \text { in dimension } d=2 \\
E_{v_{\rho}}[f] & =0 \text { in dimension } d \geq 3 .
\end{aligned}
$$

Motivated by the proposition, we will say a mean-zero function local $f$ supported on coordinates in $\Lambda_{\ell}$ has degree $n \geq 0$ if 


$$
E_{v_{\rho}}\left[f(\eta)\left(\sum_{x \in \Lambda_{\ell}} \eta(x)\right)^{n}\right] \neq 0
$$

but

$$
E_{v_{\rho}}\left[f(\eta)\left(\sum_{x \in \Lambda_{\ell}} \eta(x)\right)^{r}\right]=0 \quad \text { when } \quad r<n .
$$

Let $\widetilde{f}(y)=E_{v_{y}}[f]$. Since

$$
E_{v_{y}}[f]=\frac{1}{E_{v_{\rho}}\left[e^{\lambda(y) \eta(0)}\right]\left|\Lambda_{\ell}\right|} E_{v_{\rho}}\left[f(\eta) e^{\lambda(y) \Sigma_{x \in \Lambda_{\ell}} \eta(x)}\right]
$$

$f$ is of degree $n$ exactly when

$$
d^{n} / d y^{n} \widetilde{f}(\rho) \neq 0 \quad \text { but } \quad d^{r} / d y^{r} \widetilde{f}(\rho)=0 \quad \text { when } \quad r<n .
$$

We remark that when $p$ is symmetric, the limiting variance can be computed from monotone convergence since

$$
E_{v_{\rho}}\left[\bar{f} P_{s} \bar{f}\right]=E_{v_{\rho}}\left[\left(P_{s / 2} \bar{f}\right)^{2}\right] \geq 0
$$

then

$$
\begin{aligned}
\sigma^{2}(\rho, f) & =\lim _{t \uparrow \infty} 2 \int_{0}^{t}(1-s / t) E_{v_{\rho}}\left[\left(P_{s / 2} \bar{f}\right)^{2}\right] d s \\
& =2 \int_{0}^{\infty} E_{v_{\rho}}\left[\bar{f}\left(\eta_{0}\right) \bar{f}\left(\eta_{s}\right)\right] d s:=\sigma^{2}(\rho, f) .
\end{aligned}
$$

One can see from the formula that $\sigma^{2}(\rho, f)>0$ in the symmetric case.

To relate the limiting variance to so-called $H_{-1, \lambda, L}$ resolvent norms of $f$, define

$$
\begin{aligned}
\|f\|_{-1, \lambda, L}^{2} & :=E_{v_{\rho}}\left[\bar{f},(\lambda-L)^{-1} \bar{f}\right] \\
& =\int_{0}^{\infty} e^{-\lambda s} E_{v_{\rho}}\left[\bar{f} P_{s} \bar{f}\right] d s .
\end{aligned}
$$

Also, when the limit exists, define the $H_{-1, L}$ norm of $f$ by

$$
\|f\|_{-1, L}:=\lim _{\lambda \downarrow 0}\|f\|_{-1, \lambda, L} .
$$

In the symmetric case, when $L=S$, we will call

$$
\|f\|_{-1, \lambda}:=\|f\|_{-1, \lambda, S}
$$

and

$$
\|f\|_{-1}:=\|f\|_{-1, S} .
$$

Then, for the general process, 


$$
\begin{aligned}
\|f\|_{-1, \lambda, L}^{2} & =\lambda^{2} \int_{0}^{\infty} e^{-\lambda t} \int_{0}^{t} \int_{0}^{s} E_{v_{\rho}}\left[\bar{f} P_{u} \bar{f}\right] d u d s d t \\
& =\frac{\lambda^{2}}{2} \int_{0}^{\infty} e^{-\lambda t} \sigma_{t}^{2}(\rho, f) d t .
\end{aligned}
$$

In the symmetric case, as

$$
E_{v_{\rho}}\left[\bar{f} P_{s} \bar{f}\right]=E_{v_{\rho}}\left[\left(P_{s / 2} \bar{f}\right)^{2}\right] \geq 0
$$

we observe the limiting variance is well-defined (although possibly infinite):

$$
\begin{aligned}
\sigma^{2}(\rho, f) & =2 \int_{0}^{\infty} E_{v_{\rho}}\left[\bar{f}\left(\eta_{0}\right) \bar{f}\left(\eta_{s}\right)\right] d s \\
& =2 \lim _{\lambda \downarrow 0}\|f\|_{-1, \lambda}^{2}=2\|f\|_{-1}^{2} .
\end{aligned}
$$

We remark, in the $H_{-1}$ notation, the admissibility conditions for a function $f$ in Proposition 1 are equivalent to $\|f\|_{-1}<\infty$. Although we will not need it, but to complete the discussion, we note $\|f\|_{-1}$ is often represented (cf. Chapters 2,5 of [6]) as

$$
\|f\|_{-1}^{2}=\sup _{\phi \text { local }}\left\{\frac{E_{v_{\rho}}[\bar{f} \phi]}{D_{v_{\rho}}(\phi)^{1 / 2}}\right\}
$$

where the Dirichlet form

$$
D_{v_{\rho}}(\phi)=E_{v_{\rho}}[\phi(-S \phi)]=\frac{1}{4} \sum_{x \in \mathbb{Z}^{d}} \sum_{y \in \mathbb{Z}^{d}} E_{v_{\rho}}\left[g(\eta(x))\left(\phi\left(\eta^{x, x+y}\right)-\phi(\eta)\right)^{2}\right] s(y) .
$$

On the other hand, when $p$ is asymmetric, the limiting variance $\sigma^{2}(\rho, f)$ can be shown to exist for certain functions. We say that $f$ is coordinatewise increasing if $\eta(x) \leq \zeta(x)$ for all $x \in \mathbb{Z}^{d}$ then $f(\eta) \leq f(\zeta)$. For such a function,

$$
P_{s} f(\eta)=E[f(\eta(s)) \mid \eta(0)=\eta]
$$

is itself a coordinatewise increasing function: When $\xi \leq \zeta$ coordinatewise, let $\beta(x)=\zeta(x)-\xi(x)$ for $x \in \mathbb{Z}^{d}$. Let $\eta_{t}^{0}$ and $\eta_{t}^{1}$ be processes starting in $\xi$ and $\zeta$ respectively. Then, by the basic coupling, $\eta_{t}^{1}=\eta_{t}^{0}+\beta_{t}$ where $\beta_{t}$ follows secondclass particles. In particular, as $\bar{f}$ is increasing, with respect to the coupling measure $\widehat{P}$,

$$
P_{s} \bar{f}(\zeta)-P_{s} \bar{f}(\xi)=\widehat{E}\left[\bar{f}\left(\eta_{s}^{1}\right)-\bar{f}\left(\eta_{s}^{0}+\beta_{s}\right)\right] \geq 0 .
$$

Then, for nontrivial coordinatewise increasing functions, $E_{v_{\rho}}\left[\bar{f} P_{s} \bar{f}\right]>0$ as $v_{\rho}$, being a product measure, is a FKG measure. Therefore, the limit

$$
\sigma^{2}(\rho, f)=2 \int_{0}^{t} E_{v_{\rho}}\left[\bar{f} P_{s} \bar{f}\right] d s>0
$$

for such functions. 
For the general process, when $f$ is admissible, it is natural to ask if a functional central limit theorem holds for the diffusively scaled additive functional. When $p$ is symmetric, by the Kipnis-Varadhan CLT for reversible Markov processes, this is indeed the case $[5,15]$ and the limit in the uniform topology is a Brownian motion with diffusion coefficient $\sigma^{2}(\rho, f)$. Moreover, for nearest-neighbor systems and a class of functions $f$ in $d \leq 2$ such that $\widetilde{f}^{\prime}(\rho) \neq 0$, which are not admissible, the super-diffusive orders of $\sigma_{t}^{2}(\rho, f)$ and the limit laws of $A_{\bar{f}}(t)$ scaled by the standard deviation have also been found [9]. To give an example, in dimension $d=1$ when $E_{v_{\rho}}[f]=0$ and $E_{v_{\rho}}\left[f \sum_{x \in \Lambda_{\ell}} \eta(x)\right] \neq 0$, the limit law is a fractional Brownian motion with Hurst parameter $3 / 4$. See [9] for the full statements.

The purpose of this note is to understand the fluctuation behaviors under meanzero asymmetric and asymmetric with drift zero-range processes. When $p$ is meanzero, we will show that the generator $L$ satisfies a 'sector inequality'. As a consequence, by the method in [16], the variance behaviors in terms of orders are the same as if the process were symmetric. When $f$ is admissible, the limit

$$
\sigma^{2}(\rho, f)=\lim _{t \uparrow \infty} \frac{1}{t} E_{v_{\rho}}\left[A_{\bar{f}}(t)^{2}\right]
$$

converges, and the diffusively scaled additive functional still tends to a Brownian motion.

Theorem 1. Suppose $g$ and p satisfy assumptions (LIP), $(F R),(G)$, and in addition suppose $p$ is mean-zero. Let $f$ be a local function supported on coordinates in $\Lambda_{\ell}$. Then, $f$ is admissible if and only if the conditions in Proposition 1 are met.

In the case, $f$ is admissible, the limiting variance $\sigma^{2}(\rho, f)$ converges and we have, in the uniform topology,

$$
\lim _{\lambda \uparrow \infty} \frac{1}{\sqrt{\lambda}} A_{\bar{f}}(\lambda t) \Rightarrow \sigma(\rho, f) B_{t}
$$

where $B_{t}$ is the standard Browninan motion on $\mathbb{R}$.

Remark 1 . In the mean-zero case, for inadmissible $f$, it remains open to derive the limit laws under the appropriate scalings.

When the system is asymmetric with drift, one might have the intuition that the admissibility of a function should follow what happens in the 'transient' regime in the symmetric case. With the additional assumption of attractivity, this is indeed the case.

Theorem 2. Suppose assumptions (LIP), (INC) $(F R),(G)$ hold, and in addition suppose $p$ is asymmetric with drift. Let $f$ be a local function supported on coordinates in $\Lambda_{\ell}$. Then, $f$ is admissible if and only if $E_{v_{\rho}}[f]=0$.

When, $f$ is an increasing function, the limiting variance exists and is finite, $\sigma^{2}(\rho, f)<\infty$ and in the uniform topology

$$
\lim _{\lambda \uparrow \infty} \frac{1}{\sqrt{\lambda}} A_{\bar{f}}(\lambda t) \Rightarrow \sigma(\rho, f) B_{t} .
$$


Remark 2. When $f$ is the difference of two increasing functions, $\sigma^{2}(\rho, f)$ exists and the last statement of the theorem holds (see [13]). However, for more general $f$, in the asymmetric with drift case, this is an open question.

\section{Proof of Theorem 1: Mean-zero dynamics}

The main step is the following sector inequality, whose proof is deferred to the end of the section. Recall the definition of the Dirichlet form $D_{v_{\rho}}(\phi)$.

Proposition 2. Under the assumptions of Theorem 1, there is a constant $C=$ $C(\rho, p, d)$ such that for local functions $\phi, \psi: \Omega \rightarrow \mathbb{R}$ we have

$$
\left|E_{v_{\rho}}[\phi L \psi]\right| \leq C D_{v_{\rho}}(\phi)^{1 / 2} D_{v_{\rho}}(\psi)^{1 / 2} .
$$

When the process is symmetric, since $S$ is a nonpositive operator, a sector inequality trivially holds by Schwarz inequality and the constant $C=1$.

Proof of Theorem 1. The main argument follows the argument in [13] for meanzero simple exclusion processes, which compares $H_{-1}$ norms with respect to $L$ and the symmetrized generator $S$.

As in Lemma 4.4 of [13], we have for a constant $C_{1}>0$ that

$$
C_{1}^{-1}\|f\|_{-1, \lambda} \leq\|f\|_{-1, \lambda, L} \leq C_{1}\|f\|_{-1, \lambda} .
$$

Next, when the sector inequality in Proposition 2 holds, as computed in [16], the limit exists,

$$
\sigma^{2}(\rho, f)=\lim _{t \uparrow \infty} t^{-1} \sigma_{t}^{2}(\rho, f)=2 \lim _{\lambda \downarrow 0}\|f\|_{-1, \lambda, L}=2\|f\|_{-1, L} .
$$

Moreover,

$$
\|f\|_{-1, L}=\lim _{\lambda \downarrow 0}\|f\|_{-1, \lambda, L} \leq C_{1} \lim _{\lambda \downarrow 0}\|f\|_{-1, \lambda}=C_{1}\|f\|_{-1} .
$$

Then, given that $f$ satisfies the admissibility conditions in Theorem 1, we have

$$
\|f\|_{-1, L} \leq C_{1}\|f\|_{-1}<\infty .
$$

Conversely, suppose $f$ does not satisfy the admissibility conditions in Theorem 1 , and $\sup _{t>0} t^{-1} \sigma_{t}^{2}(\rho, f) \leq C_{2}$. Then, (1) is bounded by $C_{2} \int_{0}^{\infty} e^{-u} u d u$ uniformly in $\lambda$. Hence, $\|f\|_{-1, \lambda}$ is uniformly bounded in $\lambda$. But, a contradiction arises as then

$$
\lim _{\lambda \downarrow 0}\|f\|_{-1, \lambda}=\|f\|_{-1}<\infty,
$$

which means $f$ is admissible. Therefore, 


$$
\underset{t \uparrow \infty}{\limsup } t^{-1} \sigma_{t}^{2}(\rho, f)=\infty
$$

Finally, the functional CLT follows exactly the same proof given in [16] for mean-zero simple exclusion processes.

Proof of Proposition 2. Since $p$ is mean-zero and finite-range, it decomposes into a finite number of 'irreducible cycles' by Lemma 5.3 of [16]. That is,

$$
p=\sum_{i=1}^{r} \alpha_{i} \pi_{i}
$$

where $\pi_{i}$ places weight $1 / k$ on $k$ points $a_{1}, \ldots, a_{k}$ such that $a_{1}+\cdots+a_{k}=0$ and $y_{0}=0$,

$$
\left\{y_{\ell}=\sum_{j=1}^{\ell} a_{j}: 1 \leq \ell \leq k\right\}
$$

have no double points. We call the $B_{i}=\left\{0, y_{1}, \ldots, y_{k}\right\}$ as the $i$ th cycle . For example $a_{1}=-1, a_{2}=2$ and $a_{3}=-1$ corresponding to $y_{0}=0, y_{1}=-1, y_{2}=1$ and $y_{3}=0$ is an irreducible cycle.

Let $A_{B}$ be the zero-range process on the cycle $\left\{0, y_{1}, \ldots, y_{k}\right\}$ with jump probability $\pi_{B}$ where $\pi_{B}\left(a_{i}\right)=1 / k$ for $1 \leq i \leq k$. Then,

$$
L_{B}=\sum_{x \in \mathbb{Z}^{d}} A_{B+x}
$$

and

$$
L=\sum_{i=1}^{r} \alpha_{i} L_{B_{i}}
$$

(cf. [16][Lemma 5.4]).

It is enough to show the sector inequality with respect to $L_{B}$ for a specific cycle $B$. Indeed, if such a sector inequality holds, by a Schwarz inequality, we can write

$$
\begin{aligned}
E_{v_{\rho}}\left[\phi \sum_{i=1}^{r} \alpha_{i} L_{B_{i}} \psi\right] & =\sum_{i=1}^{r} \alpha_{i} E_{v_{\rho}}\left[\phi L_{B_{i}} \psi\right] \\
& \leq \sum_{i=1}^{r} \alpha_{i} C_{i} D_{v_{\rho}}^{i}(\phi)^{1 / 2} D_{v_{\rho}}^{i}(\psi)^{1 / 2} \\
& \leq C \sum_{i=1}^{r} \alpha_{i}\left(\frac{\varepsilon}{2} D_{v_{\rho}}^{i}(\phi)+\frac{1}{2 \varepsilon} D_{v_{\rho}}^{i}(\psi)\right) \\
& =C\left(\frac{\varepsilon}{2} \sum_{i=1}^{r} \alpha_{i} D_{v_{\rho}}^{i}(\phi)+\frac{1}{2 \varepsilon} \sum_{i=1}^{r} \alpha_{i} D_{v_{\rho}}^{i}(\psi)\right)
\end{aligned}
$$

where $D_{v_{\rho}}^{i}$ is the Dirichlet form with respect to $S_{B_{i}}$, the symmetrization of $L_{B_{i}}, C=$ $\max _{i=1, \cdots, r}\left\{C_{i}\right\}$ and $\varepsilon>0$. Taking the infimum over $\varepsilon>0$, allows to bound the left-hand side by 


$$
C D_{v_{\rho}}(\phi)^{1 / 2} D_{v_{\rho}}(\psi)^{1 / 2}
$$

Moreover, it will be enough to show the sector inequality with respect to $A_{B}$. Indeed, if so, by the same Schwarz inequality as above, we can write

$$
E_{v_{\rho}}\left[\phi \sum_{x \in \mathbb{Z}^{d}} A_{B+x} \psi\right] \leq C_{B}\left(\frac{\varepsilon}{2} \sum_{x \in \mathbb{Z}^{d}} D_{v_{\rho}}^{x}(\phi)+\frac{1}{2 \varepsilon} \sum_{x \in \mathbb{Z}^{d}} D_{v_{\rho}}^{x}(\psi)\right)
$$

where $D_{v_{\rho}}^{x}$ is the Dirichlet form with respect to symmetrization of $A_{B+x}$. Since no bond $(z, w)$ is double counted,

$$
\sum_{x \in \mathbb{Z}^{d}} D_{v_{\rho}}^{x}(\phi)=D_{v_{\rho}}^{B}(\phi)
$$

where $D_{v_{\rho}}^{B}$ is the Dirichlet form with respect to $L_{B}$.

Following the scheme in [16], we now write, with $y_{k+1}=0$, that

$$
\begin{aligned}
E_{v_{\rho}}\left[\phi A_{B} \psi\right] & =\frac{1}{k} \sum_{i=0}^{k} E_{v_{\rho}}\left[\phi(\eta) \cdot g\left(\eta\left(y_{i}\right)\right)\left(\psi\left(\eta^{y_{i}, y_{i+1}}\right)-\psi(\eta)\right)\right] \\
& =\alpha(\rho) \sum_{i=0}^{k} E_{v_{\rho}}\left[\phi\left(\eta+\delta\left(y_{i}\right)\right)\left(\psi\left(\eta+\delta\left(y_{i+1}\right)\right)-\psi\left(\eta+\delta\left(y_{i}\right)\right)\right)\right]
\end{aligned}
$$

Here, $\delta(a)$ is the configuration which puts exactly one particle at location $a$. We have used the identity

$$
E_{v_{\rho}}[g(\eta(a)) h(\eta)]=\alpha(\rho) E_{v_{\rho}}[h(\eta+\delta(a))]
$$

where $\alpha(\rho)$ is the fugacity mentioned in the introduction.

Now, since the sum

$$
\sum_{i=0}^{k} \psi\left(\eta+\delta\left(y_{i}\right)\right)-\psi\left(\eta+\delta\left(y_{i+1}\right)\right)=0,
$$

the right-hand side equals

$$
\alpha(\rho) \sum_{i=0}^{k} E_{v_{\rho}}\left[\left(\phi\left(\eta+\delta\left(y_{i}\right)\right)-\phi(\eta+\delta(0))\right)\left(\psi\left(\eta+\delta\left(y_{i+1}\right)\right)-\psi\left(\eta+\delta\left(y_{i}\right)\right)\right)\right] .
$$

Note that

$$
\begin{aligned}
& \alpha(\rho) E_{v_{\rho}}\left[\left(\phi\left(\eta+\delta\left(y_{i}\right)\right)-\phi(\eta+\delta(0))\right)^{2}\right] \\
& \leq k \sum_{i=1}^{k} E_{v_{\rho}}\left[g\left(\eta\left(y_{i}\right)\left(\phi\left(\eta^{y_{i}, y_{i+1}}\right)-\phi(\eta)\right)^{2}\right] .\right.
\end{aligned}
$$


Then, the sector inequality for $A_{B}$ follows from Schwarz inequality with a constant depending on the length of the cycle $k$.

\section{Proof of Theorem 2: Asymmetric with drift dynamics}

We will make use of the following results to prove Theorem 2.

Proposition 3. Suppose that assumption (LIP) holds and $f$ is a local function which is mean-zero, $E_{v_{\rho}}[f]=0$. Then,

$$
\sigma_{t}^{2}(\rho, f) \leq 2 t\|f\|_{-1}^{2}
$$

A proof of the proposition can be found in Appendix 1.6 of [4].

Proposition 4. Under the assumptions of Theorem 2, we have that

$$
f_{1}(\eta)=g(\eta(x))-\alpha(\rho)
$$

and

$$
f_{2}(\eta)=(g(\eta(x))-\alpha(\rho))(g(\eta(y))-\alpha(\rho)) \quad \text { for } \quad x \neq y,
$$

are admissible functions.

The proof of Proposition 4 is deferred to the end of the section.

Proof of Theorem 2. We consider cases depending on the degree of the function $f$ and dimension $d$. When $f$ is admissible for the symmetrized dynamics, that is when $\|f\|_{-1}<\infty$, by Proposition $3, \sigma_{t}^{2}(\rho, f)=O(t)$, and hence $f$ is admissible for the asymmetric model.

We now argue in the exceptional cases when $\|f\|_{-1}=\infty$ that $f$ is however still admissible for the asymmetric with drift model. It will be helpful to note that $\widetilde{f}_{1}^{\prime}(\rho)=\alpha^{\prime}(\rho)$ and $\widetilde{f}_{2}^{\prime \prime}(\rho)=2 \alpha^{\prime}(\rho)$.

Case 1. In $d=2$, if $f$ is a mean-zero degree $n=1$ function, let

$$
h=f-\frac{\widetilde{f^{\prime}}(\rho)}{\alpha^{\prime}(\rho)} f_{1} .
$$

Then, as $\widetilde{h}(\rho)=\widetilde{h}^{\prime}(\rho)=0, h$ is a degree $n \geq 2$ function. Hence, $\|h\|_{-1}<\infty$ and $h$ is admissible by Proposition 3 for the asymmetric with drift model. But, since $f_{1}$ is admissible by Proposition 4, we have

$$
\sigma_{t}^{2}(\rho, f) \leq 2 \sigma_{t}^{2}(\rho, h)+2 \sigma_{t}^{2}\left(f_{1}, \rho\right)=O(t)
$$

and therefore $f$ is admissible also.

Case 2. In $d=1$, if $f$ is a mean-zero degree $n=2$ function, consider 


$$
h=f-\frac{\widetilde{f}^{\prime \prime}(\rho)}{2\left(\alpha^{\prime}(\rho)\right)^{2}} f_{2}
$$

Since $\widetilde{h}(\rho)=\widetilde{h}^{\prime}(\rho)=\widetilde{h}^{\prime \prime}(\rho)=0, h$ is at degree $n \geq 3$ function and hence admissible. Since $f_{2}$ is also admissible (Proposition 4$), f$ is admissible by the reasoning at the end of Case 1.

On the other hand, if $f$ is a mean-zero degree $n=1$ function, consider

$$
k=f-\frac{\widetilde{f^{\prime}}(\rho)}{\alpha^{\prime}(\rho)} f_{1} .
$$

Again, as $\widetilde{k}(\rho)=\widetilde{k}^{\prime}(\rho)=0, k$ is a degree $n \geq 2$ function. By the conclusion just above, if $k$ is a degree $n=2$ function, it is admissible. If $k$ is a degree $n \geq 3$ function, it is already admissible. Since $f_{1}$ is also admissible (Proposition 4), we conclude then that $f$ is admissible.

Finally, when $f$ is an increasing coordinatewise function, the same argument as given for Theorem 1.1 of [13], making use of a Newman-Wright CLT yields the weak convergence in the theorem.

Proof of Proposition 4. We follow a technique given in [3]. We prove that $f_{2}$ is admissible. The argument for admissibility of $f_{1}$ is simpler and omitted.

Recall that $\bar{h}=h-E_{v_{\rho}}[h]$. Since $f_{2}$ is increasing, $E_{v_{\rho}}\left[f_{2} P_{s} f_{2}\right] \geq 0$ and the variance of the additive functional is bounded

$$
\sigma_{t}^{2}\left(\rho, f_{2}\right) \leq 2 t \int_{0}^{t} E_{v_{\rho}}\left[\overline { g } \left(\eta_{s}(x) \bar{g}\left(\eta_{s}(y) \cdot \bar{g}\left(\eta_{0}(x)\right) \bar{g}\left(\eta_{0}(y)\right)\right] d s .\right.\right.
$$

The integrand, using stationarity and the basic coupling, can be written as

$$
\begin{aligned}
& E_{v_{\rho}}\left[\bar{g}\left(\eta_{s}(x) \bar{g}\left(\eta_{s}(y)\right) \cdot \bar{g}\left(\eta_{0}(x)\right) \bar{g}\left(\eta_{0}(y)\right)\right]\right. \\
& =\alpha^{2}(\rho)\left\{E_{v_{\rho}}\left[P_{s} f_{2}(\eta+\delta(x)+\delta(y))\right]-E_{v_{\rho}}\left[P_{s} f_{2}(\eta)\right]\right\} \\
& =\alpha^{2}(\rho) \widehat{E}\left[g\left(\eta_{s}(x)+\xi_{s}(x)+\chi_{s}(x)\right) g\left(\eta_{s}(y)+\xi_{s}(y)+\chi_{s}(y)\right)-g\left(\eta_{s}(x)\right) g\left(\eta_{s}(y)\right)\right]
\end{aligned}
$$

where $\xi_{s}$ and $\chi_{s}$ are the processes following second-class particles starting at $x$ and $y$ respectively.

Note by (LIP) and explicit computation,

$$
\begin{aligned}
E_{v_{\rho}}\left[g^{2}(\eta(x)+2)\right] & =2 E_{v_{\rho}}\left[(g(\eta(x)+2)-g(\eta(x)))^{2}\right]+2 E_{v_{\rho}}\left[g^{2}(\eta(x))\right] \\
& \leq 2 K^{2}+2 \alpha(\rho) E_{v_{\rho}}[g(\eta(x)+1)] \\
& \leq 2 K^{2}+2 \alpha(\rho)[K+\alpha(\rho)]<\infty
\end{aligned}
$$

Then, by adding and subtracting terms and Schwarz inequality, one can bound the integrand (2) by $C \sum_{z=x, y}\left[\widehat{P}\left(\chi_{s}(z)=1\right)+\widehat{P}\left(\xi_{s}(z)=1\right)\right]$. For instance, 


$$
\left(\widehat{E}\left[g\left(\eta_{s}(z)+\xi_{s}(z)+\chi_{s}(z)\right)-g\left(\eta_{s}(z)+\xi_{s}(z)\right)\right]^{2}\right)^{1 / 2} \leq K \widehat{P}\left(\chi_{s}(z)=1\right) .
$$

To finish the proof, we now bound the integral

$$
\int_{0}^{\infty} \widehat{P}\left(\chi_{s}(x)=1\right) d s<\infty
$$

as the integrals of $\widehat{P}\left(\chi_{s}(y)=1\right)$ and $\widehat{P}\left(\xi_{s}(z)=1\right)$ for $z=x, y$ are similar.

Construct, following Kipnis's paper, the motion of the second-class particles. We follow the two particles individually $\left(\xi_{s}, \chi_{s}\right)$. From the basic coupling, the rate of the jumps from a site $x$ is given by $g\left(\eta_{s}(x)+\xi_{s}(x)+\chi_{s}(x)\right)-g\left(\eta_{s}(x)\right)$. We will suppose, if both particles are at $x$, then one of them is chosen at random to make the jump.

Let $X_{0}=y$ and $\left\{X_{i}: i \geq 1\right\}$ be the position of a random walk on $\mathbb{Z}^{d}$ according to the transient jump probability $p$. Let also $\left\{T_{i}: i \geq 1\right\}$ be independent random variables with exponential distribution with mean 1 . Then, we define jump times $\left\{\tau_{i}: i \geq 1\right\}$ and process values as follows:

$$
\begin{aligned}
\tau_{1}=\inf \{u & >0: \int_{0}^{u} \frac{\chi_{s}\left(X_{0}\right)}{\xi_{s}\left(X_{0}\right)+\chi_{s}\left(X_{0}\right)} \\
& \left.\cdot\left(g\left(\eta_{s}\left(X_{0}\right)+\xi_{s}\left(X_{0}\right)+\chi_{s}\left(X_{0}\right)\right)-g\left(\eta_{s}\left(X_{0}\right)+\xi_{s}\left(X_{0}\right)\right)\right) d s \geq T_{1}\right\} .
\end{aligned}
$$

Set $\chi_{s}(x)=\mathbf{1}_{X_{0}(x)}$ for $0 \leq s<\tau_{1}$. Also, for $r \geq 1$,

$$
\begin{aligned}
\tau_{r+1}= & \inf \left\{u>\tau_{r-1}^{u}: \int_{\tau_{r-1}}^{u} \frac{\chi_{s}\left(X_{r}\right)}{\xi_{s}\left(X_{r}\right)+\chi_{s}\left(X_{r}\right)}\right. \\
& \left.\cdot\left(g\left(\eta_{s}\left(X_{r}\right)+\xi_{s}\left(X_{r}\right)+\chi_{s}\left(X_{r}\right)\right)-g\left(\eta_{s}\left(X_{r}\right)+\xi_{s}\left(X_{r}\right)\right)\right) d s \geq T_{r+1}\right\}
\end{aligned}
$$

and $\chi_{s}(x)=\mathbf{1}_{X_{r}(x)}$ for $\tau_{r} \leq s<\tau_{r+1}$.

The dynamics for $\xi_{s}$ is similarly defined. Note that with respect to $v_{\rho}$, since $g$ is increasing,

$$
\begin{aligned}
\int_{0}^{\infty} & \frac{\chi_{s}\left(X_{r}\right)}{\xi_{s}\left(X_{r}\right)+\chi_{s}\left(X_{r}\right)}\left(g\left(\eta_{s}\left(X_{r}\right)+\xi_{s}\left(X_{r}\right)+\chi_{s}\left(X_{r}\right)\right)-g\left(\eta_{s}\left(X_{r}\right)+\xi_{s}\left(X_{r}\right)\right)\right) d s \\
\geq & \frac{1}{2} \int_{0}^{\infty} \min \left\{g\left(\eta_{s}\left(X_{r}\right)+2\right)-g\left(\eta_{s}\left(X_{r}\right)+1\right), g\left(\eta_{s}\left(X_{r}\right)+1\right)-g\left(\eta_{s}\left(X_{r}\right)\right)\right\} d s \\
\geq & \frac{1}{2} \inf _{x \in \mathbb{Z}^{d}} \int_{0}^{\infty} \min \left\{g\left(\eta_{s}(x)+2\right)-g\left(\eta_{s}(x)+1\right), g\left(\eta_{s}(x)+1\right)-g\left(\eta_{s}(x)\right)\right\} d s .
\end{aligned}
$$

By the ergodic theorem, for each $x \in \mathbb{Z}^{d}$ in the countable space $\mathbb{Z}^{d}$,

$$
\int_{0}^{\infty} \min \left\{g\left(\eta_{s}(x)+2\right)-g\left(\eta_{s}(x)+1\right), g\left(\eta_{s}(x)+1\right)-g\left(\eta_{s}(x)\right)\right\} d s=\infty \text { a.s. }
$$

Therefore, all the times $\tau_{r}$ are finite a.s. 
Then,

$$
\int_{0}^{\infty} \chi_{s}(x) d s=\sum_{j=0}^{\infty} T_{j} 1_{x}\left(X_{j}\right) .
$$

Take expectation on both sides to obtain

$$
\int_{0}^{\infty} \widehat{P}\left(\chi_{s}(x)=1\right) d s=\sum_{j=0}^{\infty} P\left(X_{j}=x\right)<\infty
$$

since $\left\{X_{j}\right\}$ is transient.

\section{Acknowledgements}

The authors thank the enjoyable hospitality of CMAT (Portugal) where the conference "Particle systems and Partial Differential Equations" December 5-7, 2012 took place and part of this work was discussed.

C.B. acknowledges the support of the French Ministry of Education through the grant ANR-10-BLAN 0108 (SHEPI).

P.G. thanks FCT (Portugal) for support through the research project "NonEquilibrium Statistical Physics" PTDC/MAT/109844/2009. P.G. thanks the Research Centre of Mathematics of the University of Minho, for the financial support provided by "FEDER" through the "Programa Operacional Factores de Competitividade COMPETE” and by FCT through the research project PEst-C/MAT/UI0013/2011.

C.B. and P.G. are grateful to Égide and FCT for the research project FCT/1560/25/1/2012/S.

The research of S.S. was supported in part by NSF DMS-1159026.

\section{References}

1. Andjel, E.D. Invariant measures for the zero range process. Ann. Probab., 10, 525-547 (1982).

2. Evans, M.R., Hanney, T.: Nonequilibrium statistical mechanics of the zero-range process and related models, J. Phys. A: Math. Gen., 38, 195-240 (2005).

3. Kipnis, C.: Central Limit Theorems for Infinite Series of Queues and Applications to Simple Exclusion, Ann. Probab., 14, 397-408, (1986).

4. Kipnis, C., Landim, C.: Scaling limits of interacting particle systems, Grundlehren der Mathematischen Wissenschaften 320 Springer-Verlag, Berlin (1999).

5. Kipnis, C., Varadhan, S. R. S.: Central limit theorem for additive functionals of reversible markov processes, Commun. Math. Phys., 104, 1-19, (1986).

6. Komorowski, T. and Landim, C. and Olla, S.: Fluctuations in Markov processes, SpringerVerlag, New-York (2012).

7. Landim, C.; Sethuraman, S.; Varadhan, S.: Spectral gap for zero-range dynamics, Ann. Probab., 24, 1871-1902 (1996).

8. Liggett, T.: Interacting Particle Systems, Springer-Verlag, New York (1985). 
9. Quastel, J.; Jankowski, H.; Sheriff, J.: Central limit theorem for zero-range processes, Special issue dedicated to Daniel W. Stroock and Srinivasa S. R. Varadhan on the occasion of their 60th birthday, Methods Appl. Anal., 9, no. 3, 393-406 (2002).

10. Morris, B.: Spectral gap for the zero-range process with constant rate, Ann. Probab., 34, 1645-1664 (2006).

11. Nagahata, Y.: Spectral gap for zero-range processes with jump rate $g(x)=x^{\gamma}$, Stoch. Proc. Appl., 120, 949-958 (2010).

12. Quastel, J.: Diffusion of color in the simple exclusion process, Comm. Pure Appl. Math., 45, 623-679 (1992).

13. Sethuraman, S.: Central Limit Theorems for Additive Functionals of the Simple Exclusion Process, Ann. Probab., 28, 277-302; Correction (2006) 34, 427-428 (2000).

14. Sethuraman, S.: On extremal measures for conservative particle systems, Ann. Inst. Henri Poincare Prob. et Statistiques, 37, 139-154 (2001).

15. Sethuraman, S. and Xu, L.: A central limit theorem for reversible exclusion and zero-range particle systems, Ann. Probab., 24, 1842-1870 (1996).

16. Varadhan, S. R. S.: Self-diffusion of a tagged particle in equilibrium for asymmetric mean zero random walk with simple exclusion, Ann. Inst. Henri Poincare Prob. et Statistiques, 31, 273-285 (1995). 